\title{
Entanglement and purity loss for the system of two 2-level atoms in the presence of the Stark shift
}

\author{
Hosny A. Hessian • M. Hashem
}

Received: 21 January 2010 / Accepted: 19 October 2010 / Published online: 8 December 2010

(C) The Author(s) 2010. This article is published with open access at Springerlink.com

\begin{abstract}
In this paper, we have presented an analytic solution for two 2-level identical atoms interacting with a single-mode quantized radiation field, taking into account the level shifts produced by Stark shift. We assume that the two atoms are initially prepared in the exited state and the field in a coherent state. We investigate the purity loss of the system and bipartite partitions of the system. The effects of the Stark shift on the purity loss of the system and different bipartite partitions of the system (field-two atoms, atom-(field+atom)) through the tangles are considered. In particular, the effect of the Stark shift on the amount of entanglement between atoms and field is evaluated by the negativity.
\end{abstract}

\section{Introduction}

The interaction between a two-level atom and the radiation field is a quantum optical problem that lies at the heart of many problems in laser physics and quantum optics. The fundamental difference between quantum and classical physics is the possible existence of nonclassical correlations between distinct quantum systems. The physical property responsible for the nonclassical correlations is called entanglement. From the beginning of the 1990s, the field of quantum information theory opened up and expanded rapidly. A number of entanglement measures have been discussed in the literature, such as the von Neumann reduced entropy and the relative entropy of entanglement [1]. The quantum entropy has been used to study the dynamic characteristics of a 2-level atom interacting with a light field [2]. It is shown that the quantum entropy is a useful physical quantity for measuring the degree of the entanglement of the state. On the other hand, quantum entanglement is one of the most

H. A. Hessian $(\varangle) \cdot$ M. Hashem

Faculty of Science, Assiut University, Assiut, Egypt

e-mail: ammar_67@yahoo.com 
profound features of quantum mechanics and has been considered to be a valuable physical resource in the new field of quantum information science, including quantum computation [3], quantum teleporation [4], quantum dense coding [5], and quantum information processing [6]. Therefore, the study of quantum entanglement is very significant.

One of the particular interesting schemes in which entanglement can be created is a system containing two two-level atoms, since they can represent two qubits, the building blocks of the quantum gates that are essential to implement quantum protocols in quantum information processing. A number of theoretical studies have been performed on preparation of a two-atom system in an entangled state. Two-atom entangled states have been demonstrated experimentally using ultra cold trapped ions $[7,8]$ and cavity quantum electrodynamics schemes [9]. As a typical model, the TavisCummings model (TCM) [10] can be used to understand the evolution of entanglement in its different bipartite partitions. The two-atom TCM describes the simplest fundamental interaction between a single mode of the quantized electromagnetic field and two atoms under the usual two-level and rotating wave approximations. A thorough understanding of the dynamical evolution of entanglement of the TCM has obvious implications for the performance of quantum information processing [1113] as well as for our understanding of fundamental quantum mechanics [11]. Some authors investigated the properties of the quantum entanglement in the two atoms [14-17].

Entanglement between two particles can be identified by calculating, for example, the Wootters entanglement measure (concurrence) $[18,19]$, or a measure proposed by Peres [20] and Horodecki [21] given in terms of the negative eigenvalues (negativity) of the partial transposition of the density matrix of the two particle system. The tangle for each of the bipartite partitions is calculated by Tessier et al. [13] for the case of the two-atom TCM. The inherent tripartite correlations in the system is also studied by using the so-called residual tangle. Angelo et al. [22] analyzed the entanglement of the quantum subsystems in TCM, using the reduceddensity linear entropy. But the study of tripartite in TCM is still an open issue. Most of the authors who have treated this problem have dealt with the case in which the Stark shift has been ignored [23-25]. However, in reality it cannot be ignored. The authors in [26] discuss the same model in the case of one photon, but our interest lies in the case where the Stark shift and two photon are included.

These important topics previously discussed are the motivation to study simultaneously the evolution of two identical 2-level atoms interacting with a single-mode quantized radiation field, taking into account the level shifts produced by Stark shift. For chosen initial state, we investigate the von Neumann entropy, as a measure of purity loss and bipartite partitions of the system (tangles). The effects of the Stark shift on the purity loss of the system and the tangles are considered. In particular, the effect of the Stark shift on the amount of entanglement between atoms and field is evaluated by the negativity. This paper is arranged as follows: Section 2 is devoted to the physical system and its dynamics. In Sect. 3, we employ the analytical results obtained in Sect. 2 to discuss the von Neumann entropy,tangles and negativity. Finally in Sect. 4, we present our conclusion. 


\section{The model and its solution}

Tow-photon transitions in atomic systems play an important role because of high degree of correlation between emitted photons[33] which leads to non-classical behavior of emitted light. We consider the interaction of a two identical 2-level atoms with a single mode quantized electromagnetic field. In this system, either atom can transit from exited state $|+\rangle$ to ground state $|-\rangle$ under the driving of a resonant field and emit 2 photons. Also, either atom that is in state $|-\rangle$ can absorb such 2 photons and jump to state $|+\rangle$. For simplicity, we consider the case in which the atoms and the field are exactly resonant and the two atom makes two-photon transitions of frequency $2 \omega$ between the nondegenerate state $|g\rangle$ (the ground state, energy $E_{g}$ ) and $|e\rangle$ (the exited state, energy $E_{e}$ ) see Fig. 1. The transitions are mediated by a single intermediate level $|i\rangle$ (energy $E_{i}$, with $E_{e}>E_{i}>E_{g}$ ): the frequencies for $|g\rangle \rightarrow|i\rangle$ and $|i\rangle \rightarrow|e\rangle$ are $\omega-\Delta=E_{i}-E_{g}$ and $\omega+\Delta=E_{e}-E_{i}$, respectively. In the rotating wave approximation, the effective Hamiltonian of the system takes the form $(\hbar=1)$ [26]:

$$
\begin{aligned}
\hat{H}=\omega & \left(\hat{a}^{\dagger} \hat{a}+\frac{1}{2}\right)+\omega\left(\hat{\sigma}_{1}^{3}+\hat{\sigma}_{2}^{3}\right)+g \sum_{i=1}^{2}\left(\hat{a}^{2} \hat{\sigma}_{i}^{+}+\hat{a}^{\dagger^{2}} \hat{\sigma}_{i}^{-}\right) \\
& +\hat{a}^{\dagger} \hat{a} \beta_{1}\left(\hat{\sigma}_{1}^{-} \hat{\sigma}_{1}^{+}+\hat{\sigma}_{2}^{-} \hat{\sigma}_{2}^{+}\right)+\hat{a}^{\dagger} \hat{a} \beta_{2}\left(\hat{\sigma}_{1}^{+} \hat{\sigma}_{1}^{-}+\hat{\sigma}_{2}^{+} \hat{\sigma}_{2}^{-}\right),
\end{aligned}
$$

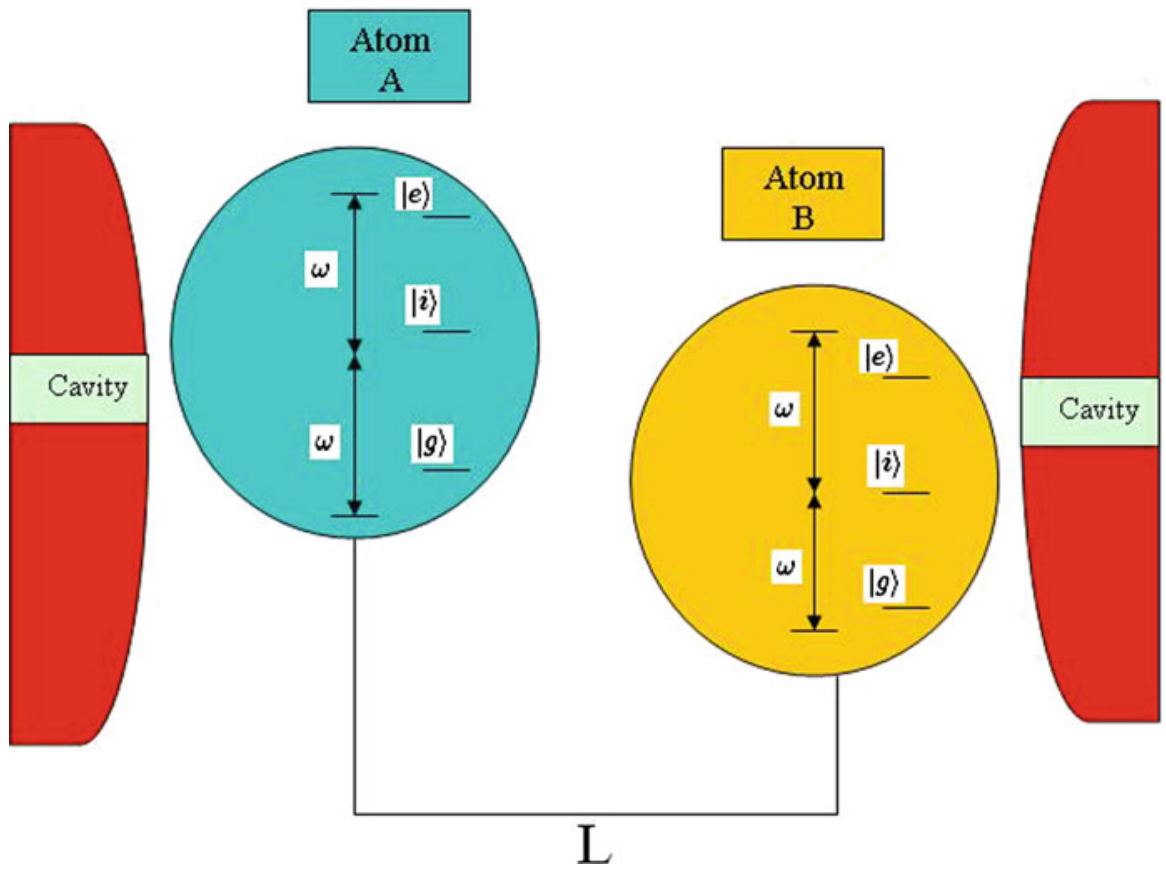

Fig. 1 A diagram depicting two two-level atoms of variable spatial separation interacting with a singlemode radiation cavity field through a two-photon process. The first atom is fixed at the peak of cavity field, while the second atom can experience variable coupling at a distance L. We assume the ground and exited states of the atoms have identical parity where as all intermediate levels are sufficiently non resonant that they acquire no appreciable population and are consequently located as to give rise to a significate Stark shift 
where $\hat{a}, \hat{a}^{\dagger}$ are annihilation and creation operators of the cavity field and the 2-level atoms are described by the atomic pseudospin operators $\hat{\sigma}_{i}^{3}, \hat{\sigma}_{i}^{ \pm}$. Each atom contains three-level $(|g\rangle,|i\rangle,|e\rangle)$ where the coupling constants $g_{1}$ (for $|g\rangle \rightarrow|i\rangle$ ), $g_{2}$ (for $|i\rangle \rightarrow|e\rangle$ ) and $\Delta$ determine the Stark-shift parameters $\beta_{1}$ and $\beta_{2}$ of the two levels and also the coupling $g$ between the effective two levels atom, states $|g\rangle$ and $|e\rangle$, and the field mode:

$$
\beta_{1}=\frac{g_{1}^{2}}{\triangle}, \quad \beta_{2}=\frac{g_{2}^{2}}{\triangle}, \quad g=\frac{g_{1} g_{2}}{\triangle}
$$

It is clear that for $g_{1}$ comparable with $g_{2}, \beta_{1}, \beta_{2}$, and $g$ are comparable with one another, and the Stark-shift can not be ignored. Now we can generalize the Hamiltonian to the case with arbitrary $k$ photon:

$$
\begin{aligned}
\hat{H}= & \omega\left(\hat{a}^{\dagger} \hat{a}+\frac{1}{2}\right)+\omega\left(\hat{\sigma}_{1}^{3}+\hat{\sigma}_{2}^{3}\right)+g \sum_{i=1}^{2}\left(\hat{a}^{k} \hat{\sigma}_{i}^{+}+\hat{a}^{\dagger} \hat{\sigma}_{i}^{-}\right) \\
& +\hat{a}^{\dagger} \hat{a} \beta_{1}\left(\hat{\sigma}_{1}^{-} \hat{\sigma}_{1}^{+}+\hat{\sigma}_{2}^{-} \hat{\sigma}_{2}^{+}\right)+\hat{a}^{\dagger} \hat{a} \beta_{2}\left(\hat{\sigma}_{1}^{+} \hat{\sigma}_{1}^{-}+\hat{\sigma}_{2}^{+} \hat{\sigma}_{2}^{-}\right),
\end{aligned}
$$

The initial state of the total atom-atom field system can be written as:

$$
|\psi(0)\rangle=|\psi(0)\rangle_{F} \otimes|\psi(0)\rangle_{A}=\sum_{n=0} q_{n}|n,+,+\rangle
$$

which means that each atom starts in its exited state, the field is assumed to be initially in a coherent state, where $q_{n}=e^{-\left|\alpha^{2}\right| / 2} \frac{\alpha^{n}}{\sqrt{n !}}, \alpha=|\alpha| e^{i \phi}, \bar{n}=|\alpha|^{2}$ is the mean photon number of the coherent field and $\phi$ is the phase angle of the coherent field (here we take $\phi=0$ ). The analytical solution of the time-dependent Schrödinger equation is provided in [33] without Stark-shift. Now we can easily introduce the solution of the Schrödinger equation with Stark-shift in the interaction picture, i.e., the wave function of the system at any time $t>0$ is given by:

$$
\begin{aligned}
|\Psi(\tau)\rangle= & \sum_{n=0}^{\infty} q_{n}\left[c_{1}(n, \tau)|n,+,+\rangle+c_{2}(n, \tau)|n+k,+,-\rangle\right. \\
& \left.+c_{3}(n, \tau)|n+k,-,+\rangle+c_{4}(n, \tau)|n+2 k,-,-\rangle\right] .
\end{aligned}
$$

Then we get the explicit forms for the dynamical coefficients $c_{j}(n, \tau),(j=1,2,3,4)$ as:

$$
\begin{aligned}
& c_{1}(n, \tau)=l_{11} e^{i \alpha_{n}^{1} \tau}+l_{12} e^{i \alpha_{n}^{2} \tau}+l_{13} e^{i \alpha_{n}^{3} \tau}, \\
& c_{2}(n, \tau)=c_{3}(n, \tau)=l_{21} e^{i \alpha_{n}^{1} \tau}+l_{22} e^{i \alpha_{n}^{2} \tau}+l_{23} e^{i \alpha_{n}^{3} \tau}, \\
& c_{4}(n, \tau)=l_{31} e^{i \alpha_{n}^{1} \tau}+l_{32} e^{i \alpha_{n}^{2} \tau}+l_{33} e^{i \alpha_{n}^{3} \tau},
\end{aligned}
$$


where

$$
\begin{aligned}
l_{1 u}= & \frac{\left(\alpha_{n}^{u}+B\right)\left(\alpha_{n}^{u}+C\right)-2 \eta^{2}}{\left(\alpha_{n}^{u}-\alpha_{n}^{v}\right)\left(\alpha_{n}^{u}-\alpha_{n}^{w}\right)}, \\
l_{2 u}= & \frac{-\zeta\left(\alpha_{n}^{u}+C\right)}{\left(\alpha_{n}^{u}-\alpha_{n}^{v}\right)\left(\alpha_{n}^{u}-\alpha_{n}^{w}\right)} \\
l_{3 u}= & \frac{2 \zeta \eta}{\left(\alpha_{n}^{u}-\alpha_{n}^{v}\right)\left(\alpha_{n}^{u}-\alpha_{n}^{w}\right)}, \\
& u, v, w=1,2,3 ; \quad u \neq v \neq w
\end{aligned}
$$

with

$$
\begin{aligned}
\alpha_{n}^{1} & =2\left(-\frac{\lambda_{n}}{6}+z^{\frac{1}{3}} \cos \left(\frac{\delta}{3}\right)\right) \\
\alpha_{n}^{2} & =2\left(-\frac{\lambda_{n}}{6}+z^{\frac{1}{3}} \cos \left(\frac{\delta+2 \pi}{3}\right)\right) \\
\alpha_{n}^{3} & =2\left(-\frac{\lambda_{n}}{6}+z^{\frac{1}{3}} \cos \left(\frac{\delta+4 \pi}{3}\right)\right) \\
z & =\sqrt{x^{2}+y^{2}}, \quad \delta=\tan ^{-1} \frac{y}{x} \\
x & =\frac{27 v_{n}-9 \lambda_{n} \mu_{n}-2 \lambda_{n}^{3}}{54}, \\
y & =\frac{\sqrt{4\left(3 \mu_{n}+\lambda_{n}^{2}\right)^{3}-\left(27 v_{n}-9 \lambda_{n} \mu_{n}-2 \lambda_{n}^{3}\right)^{2}}}{54}
\end{aligned}
$$

and

$$
\begin{aligned}
\lambda_{n} & =A+B+C, \quad \mu_{n}=2\left(\zeta^{2}+\eta^{2}\right)-(A B+A C+B C), \\
v_{n} & =2\left(\zeta^{2} A+\eta^{2} C\right)-A B C, \\
\eta & =\sqrt{\frac{(n+2 k) !}{(n+k) !}}, \quad \zeta=\sqrt{\frac{(n+k) !}{(n) !}} \\
A & =\frac{2 n}{R} \quad B=\frac{\left(1+R^{2}\right)(n+k)}{R}, \quad C=2 R(n+2 k),
\end{aligned}
$$

where $R=\sqrt{\frac{\beta_{1}}{\beta_{2}}}, g=\sqrt{\beta_{1} \beta_{2}}, \beta_{1} \neq 0, \beta_{2} \neq 0$ and $\tau=g t$. The purity loss and entanglement for this system are discussed in the next section using the above results.

At any time $t>0$ the reduced two atomic density operator for the system is given by:

$$
\hat{\rho_{A}}(t)=\operatorname{Tr}_{F}[\hat{\rho}(t)]
$$


and we can write $\hat{\rho_{A}}(t)$ in the flowing form:

$$
\hat{\rho_{A}}(t)=\left(\begin{array}{llll}
x_{11} & x_{12} & x_{13} & x_{14} \\
x_{21} & x_{22} & x_{23} & x_{24} \\
x_{31} & x_{32} & x_{33} & x_{34} \\
x_{41} & x_{42} & x_{43} & x_{44}
\end{array}\right),
$$

where

$$
\begin{aligned}
x_{11} & =\sum_{n, m}^{\infty} q_{n, m} c_{1}(n, \tau) c_{1}^{*}(m, \tau) \\
x_{22(33)} & =\sum_{n, m}^{\infty} q_{n-k, m-k} c_{2(3)}(n-k, \tau) c_{2(3)}^{*}(m-k, \tau) \\
x_{44} & =\sum_{n, m}^{\infty} q_{n-2 k, m-2 k} c_{4}(n-2 k, \tau) c_{4}^{*}(m-2 k, \tau) \\
x_{12(13)} & =\sum_{n, m}^{\infty} q_{n-k, m} c_{1}(n-k, \tau) c_{2(3)}^{*}(m, \tau)=x_{21(31)}^{*} \\
x_{14} & =\sum_{n, m}^{\infty} q_{n, m-2 k} c_{1}(n-2 k, \tau) c_{4}^{*}(m, \tau)=x_{41}^{*} \\
x_{23}= & \sum_{n, m}^{\infty} q_{n-k, m-k} c_{2}(n-k, \tau) c_{3}^{*}(m-k, \tau)=x_{32}^{*} \\
x_{24(34)}= & \sum_{n}^{\infty} q_{n-2 k, m-k} c_{2(3)}(n-2 k, \tau) c_{4}^{*}(m-k, \tau) \\
= & x_{42(43)}^{*}
\end{aligned}
$$

The eigenvalues of the reduced two atomic density operator $\hat{\rho_{A}}(t)$ can be obtained as follows:

$$
\begin{aligned}
& \pi_{A}^{1}(t)=0, \quad \pi_{A}^{2}(t)=2\left[\frac{1}{6}+r^{\frac{1}{3}} \cos \left(\frac{\theta}{3}\right)\right] \\
& \pi_{A}^{3}(t)=2\left[\frac{1}{6}+r^{\frac{1}{3}} \cos \left(\frac{\theta+2 \pi}{3}\right)\right], \\
& \pi_{A}^{4}(t)=2\left[\frac{1}{6}+r^{\frac{1}{3}} \cos \left(\frac{\theta+4 \pi}{3}\right)\right]
\end{aligned}
$$

where $r=\frac{\sqrt{\Delta+2-9 \epsilon-27 \varepsilon}}{54}, \theta=\tan ^{-1} \frac{\Delta}{2-9 \epsilon-27 \varepsilon}, \Delta=4(3 \epsilon-1)^{3}+(-2+9 \epsilon+27 \varepsilon)^{2}$ with

$$
\epsilon=\sum_{i, j=1}^{4} \sum_{i<j}^{4} x_{i i} x_{j j}-\sum_{i, j=1}^{4} \sum_{i<j}^{4}\left|x_{i j}\right|^{2}
$$


and

$$
\begin{aligned}
\varepsilon= & -\sum_{i=1}^{4} x_{i i} \sum_{j<k: j, k>i}^{4} x_{j j} x_{k k}+\sum_{i=1}^{4} x_{i i} \sum_{j<k: j, k \neq i}^{4}\left|x_{j k}\right|^{2} \\
& -\sum_{i \neq j \neq k: j, k>i}^{4} x_{i j} x_{j k} x_{k i}
\end{aligned}
$$

\section{Measurement of entanglement degree}

\section{1 von Neumann entropy}

We use the von Neumann entropy as a measure of the degree of purity loss of the subsystem. The entropy of the subsystem can be defined through their respective reduced density matrix as [27]:

$$
S_{i}(t)=-\operatorname{Tr}_{i}\left(\hat{\rho}_{i}(t) \ln \hat{\rho}_{i}(t)\right), \quad(i=A \quad \text { or } \quad F)
$$

we can see that the reduced entropies of the two subsystems are identical, namely $S_{A}(t)=S_{F}(t)$. Consequently we need only to calculate the atomic reduced entropy $S_{A}(t)$. We can express the reduced entropy $S_{A}(t)$ of the two atoms in terms of the eigenvalues of $\hat{\rho_{A}}(t)$ given by (Eq. 10):

$$
S_{A}(t)=-\sum_{j=1}^{4} \pi_{A}^{j}(t) \ln \left[\pi_{A}^{j}(t)\right]
$$

It reflect the degree of the entanglement between the two atoms and the coherent field. If $S_{A}(t)$ takes its minimum value 0 , the coherent field and the two atoms are in a disentangled state. If $S_{A}(t)$ takes its maximum value, the coherent field and the two atoms are in the maximum entangled state.

In Fig. 2, we have plotted the entropy of the two atoms for two-photon $(k=2)$ case with various values of the Stark shift parameter $R$ taking the initial mean photon number $\bar{n}=100$. In the absence of the Stark shift, corresponding to the evolution of the atomic entropy in the standard two photon. It is observed that the atom entropy evolves with a period $\pi$, when $\tau=n \pi(n=0,1,2, \ldots), S_{A}(t)$ evolves to zero and the field is completely disentangled from the atom, while for $\tau=(2 n-1) \frac{\pi}{4}, S_{A}(t)$ evolves to the maximum value and the field is strongly entangled with the atoms. To see the influence of the Stark shift on the evolution of the atom entropy and purity, we plotted the atomic entropy for different values of the Stark shift. In Fig. 2b, we put the Stark shift parameter $R$ equal $1\left(\beta_{1}=\beta_{2}\right)$, which corresponds to the case in which the two levels of the atoms are equally strongly coupled with the intermediate relay level. By comparing Fig. 2a, b, we see that the evolution of the entropy is almost similar in both cases. These results correspond to the fact that the Stark shift creates an effective intensity dependent detuning $\Delta_{N}=\beta_{1}-\beta_{2}$ [28]. When $R=1\left(\beta_{1}=\beta_{2}\right), \Delta_{N}=0$. 

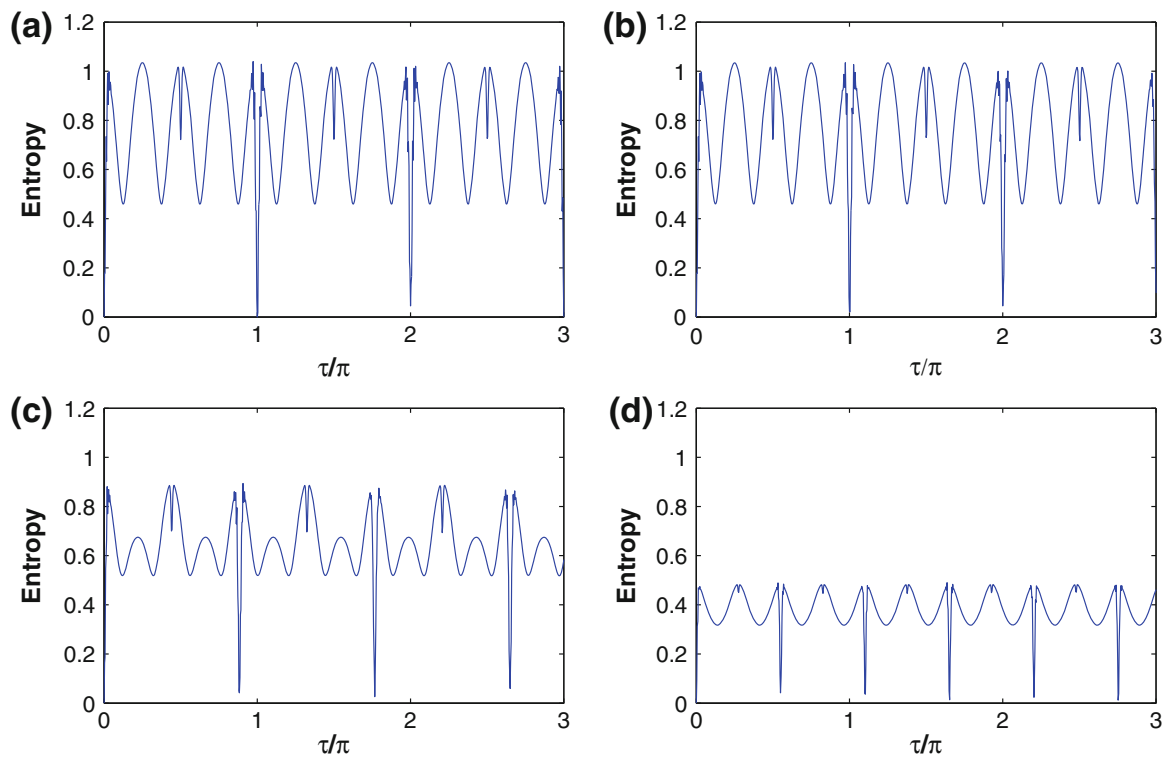

Fig. 2 The time evolution of the von Neumann entropy for two atoms prepared initially in the exited state and the field in a coherent state for two-photon $(k=2)$ with initial mean photon number $\bar{n}=100$ for various values of the Stark shift parameter $R$ : a neglect of the Stark shift $\mathbf{b} R=1\left(\beta_{1}=\beta_{2}\right) \mathbf{c} R=0.6(\mathbf{d})$ $R=0.3$

In this case, the Stark shift does not affect the time evolution of the atomic entropy. In Fig. 2c, d, we show the cases in which the two levels have unequal Stark shifts $(R<1$, in Fig. 2c $R=0.6$ while in Fig. $2 d R=0.3$ ). We see that the Stark shift leads to a decrease in the values of the maximum atomic entropy and the evolution period of the atomic entropy decreases with the parameter $R$. This can be interpreted as follows: When the parameter $R$ is reduced, the effective detuning $\Delta_{N}$ increases. This increase leads to a disentanglement between the atoms and field (the values of maximum atom entropy are reduced) [29].

\subsection{Bipartite tangles in the two atoms}

Let the two atoms in the ensemble be denoted by $A_{1}$ and $A_{2}$, respectively, and the field, or quantum bus, by F. Because of the assumed exchange symmetry, there are four nonequivalent partitions of the two atoms TCM into tensor products of bipartite subsystems: ( $i$ ) the field times the two-atoms ensemble, $F \otimes\left(A_{1} A_{2}\right)$; $(i i)$ one atom times the remaining atom and the field, $A_{1} \otimes\left(A_{2} F\right) \equiv A_{2} \otimes\left(A_{1} F\right) ;($ iii $)$ the two atoms taken separately, having traced over the field, $A_{1} \otimes A_{2}$; and (iv) one of the atoms times the field, having traced over the other atom, $A_{1} \otimes F \equiv A_{2} \otimes F$. For the special case, we calculate the tangle of the partition $(i)$ to discuss the degree of entanglement for the system under consideration. 
The tangle between two qubits in an arbitrary state is defined in terms of the concurrence $[18,19]$. Rungta et al. extended this formalism by introducing an analytic form for the concurrence of a bipartite system $A B$, with arbitrary dimensions $D_{A}$ and $D_{B}$, in an overall pure state [30]. Coffman et al. [31] defined the tangle $\tau_{2}$ for a system of two qubits as:

$$
\tau_{2}(\rho)=\max \left[0, \lambda_{1}-\lambda_{2}-\lambda_{3}-\lambda_{4}\right]^{2} .
$$

where $\lambda_{i}$ are the eigenvalues of the matrix $\hat{\rho_{A}(t)} \tilde{\rho}_{A}(t)$ with $\tilde{\rho}_{A}(t)=\left(\sigma_{y} \otimes \sigma_{y}\right) \rho_{A}^{*}\left(\sigma_{y} \otimes\right.$ $\left.\sigma_{y}\right)$ and $\sigma_{y}$ is the Pauli matrix.

Indeed, this definition can be extended directly to the result of Rungta et al. [30] in order to get an analytic form for the tangle $\tau$ of a bipartite system in a pure state with arbitrary subsystem dimensions.

$$
\tau\left(\psi_{A B}\right)=2 v_{A} v_{B}\left(1-\operatorname{Tr}\left(\rho_{A}^{2}\right)\right) .
$$

Where $v_{A}$ and $v_{B}$ are arbitrary scale factors.

Field-ensemble and one-atom-remainder tangles Under the assumption that the system is in an overall pure state, we may easily calculate the tangles in partition (i) and (ii) above by applying (Eq. 19), with $v_{A}=v_{B}=1$. Specifically,

$$
\tau_{F\left(A_{1} A_{2}\right)}=2\left[1-\operatorname{Tr}\left(\rho_{F}^{2}\right)\right]
$$

and

$$
\tau_{A_{1}\left(A_{2} F\right)}=2\left[1-\operatorname{Tr}\left(\rho_{A_{1}}^{2}\right)\right]
$$

where we have used the fact the (non-zero) eigenvalue spectra of the two marginal density operators for a bipartite division of a pure state are identical [11,32] in obtaining the rightmost equalities. The tangle has implications for the quantum control of atomic ensembles. Because the overall system is pure, any correlation between the field and the ensemble is necessarily in one-to-one correspondence with the amount of entanglement between these two subsystems. The quantum backaction on the ensemble due to measurement of the field is thus quantified by (Eq. 20). Alternatively, a measurement of one atom leads to backaction on the remaining subsystem as described by (Eq. 21).

In Fig. 3, we have plotted the tangle $\tau_{F\left(A_{1} A_{2}\right)}$ with the same parameter as in Fig. 2, we have obtained the same information about entanglement as the atomic entropy but the difference between them in the maximum and minimum value. If we put $k=1$ (one photon), and in the absence of the Stark shift corresponding to the tangle $\tau_{F\left(A_{1} A_{2}\right)}, \tau_{F\left(A_{1} A_{2}\right)}$ in the two atom TCM obtained by Ref.[13].

In Fig. 4, we have plotted the tangle $\tau_{A_{1}\left(A_{2} F\right)}$ for the two-photon for various values of the Stark shift parameter $R$. In the absence of the Stark shift, it is observed that $\tau_{A_{1}\left(A_{2} F\right)}$ evolves with a period $\pi$, when $\tau=n \pi(n=0,1,2,3, \ldots), \tau_{A_{1}\left(A_{2} F\right)}$ evolves to zero and the state is completely disentangled. on the other hand the tangle, $\tau_{A_{1}\left(A_{2} F\right)}$ 

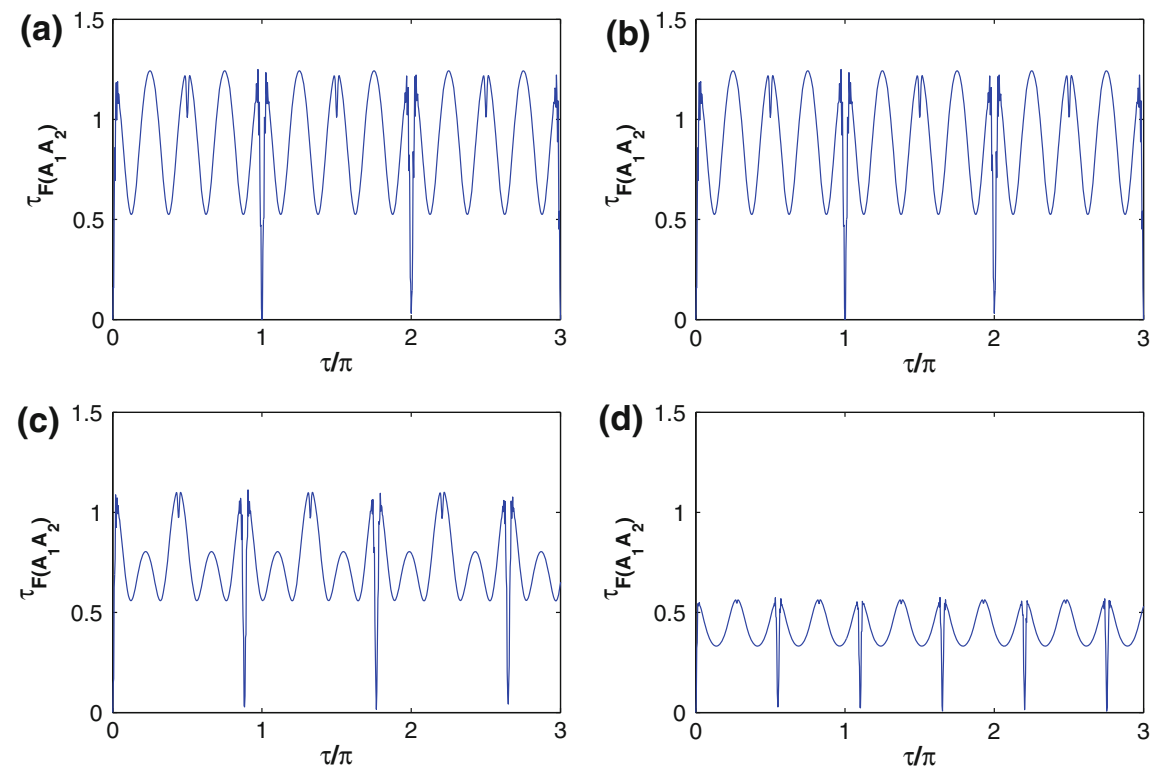

Fig. 3 Plots of the tangle $\tau_{F\left(A_{1} A_{2}\right)}$ for the two atoms are prepared initially in the exited state and the field in a coherent state for two-photon $(k=2)$ with initial mean photon number $\bar{n}=100$ for various values of the Stark shift parameter $R$ : a neglect of the Stark shift $\mathbf{b} R=1\left(\beta_{1}=\beta_{2}\right) \mathbf{c} R=0.6 \mathbf{d} R=0.3$
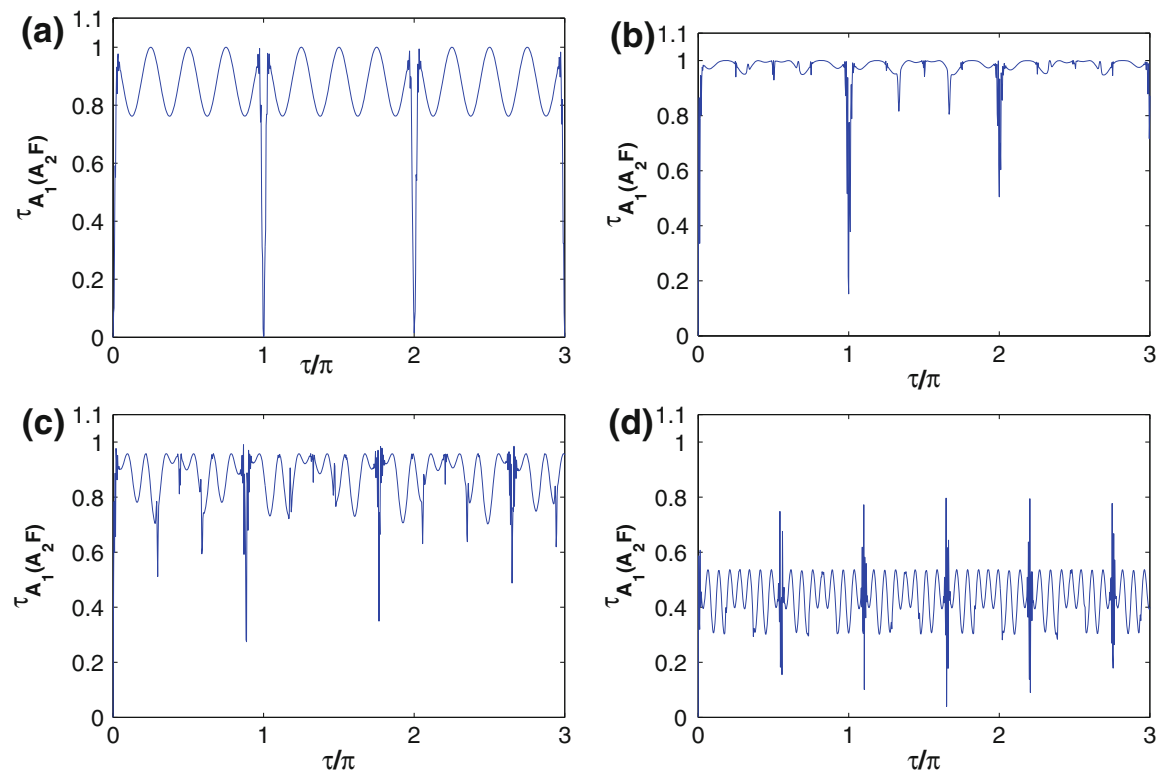

Fig. 4 Plots of the tangle $\tau_{A_{1}\left(A_{2} F\right)}$ for the two atoms are prepared initially in the exited state and the field in a coherent state for two-photon $(k=2)$ with initial mean photon number $\bar{n}=100$ for various values of the Stark shift parameter $R$ : a neglect of the Stark shift $\mathbf{b} R=1\left(\beta_{1}=\beta_{2}\right) \mathbf{c} R=0.6 \mathbf{d} R=0.3$ 
evolves to the maximum value 1 , and the state is strongly entangled see Fig. 4a. In the presence of the Stark shift, i.e. $R=1$, see Fig. $4 \mathrm{~b}$ as seen from this figure, the contribution to the purity loss of the global system is from the interaction between atom and field. Here, the tangle is used to measure the coherence loss of the atomic and field states.

In Fig. 4c, d, we show the cases in which the two levels have unequal Stark shifts ( $R<1$, in Fig. 4c $R=0.6$ while in Fig. $4 d R=0.3$ )., for this case a drastic change occurs in the $\tau_{A_{1}\left(A_{2} F\right)}$ behavior where we note an increase in the fluctuation number and consequently more periods at different intervals. Furthermore the tangle in this case shows rapid fluctuations with a slight interference between the patterns at different periods (see Fig. 4c). Finally the Stark shift leads to irregularity, purity loss and a decreasing in the values of the maximum tangle.

\subsection{Negativity}

The entanglement created by spontaneous emission is illustrated most clearly if one assumes that a system of two atoms decays spontaneously from initially unentangled (uncorrelated) states. several different measures have been proposed to identify entanglement between two atoms, and we chose the Peres-Horodecki (negativity) measure for entanglement [20,21]. The negativity criterion is given by the quantity

$$
E=\max \left(0,-2 \sum_{i} \mu_{i}\right),
$$

where the sum is taken over the negative eigenvalues $\mu_{i}$ of the partial transposition of the density matrix $\rho$ of the system. The value $\mathrm{E}=1$ corresponds to maximum entanglement between the atoms while $\mathrm{E}=0$ describes completely separated atoms.

Dynamics of negativity is presented in Fig. 5 for the two atoms are initially prepared in exited state $|+,+\rangle$ and the field in a coherent state. We have plotted the negativity $E$ for various values of the Stark shift parameter $R$. In the absence of the Stark shift (see Fig. 5a), it is observed that $E$ evolves with a period $\pi$, when $\tau=n \pi(n=0,1,2, \ldots)$, $E$ evolves to zero and the state is completely disentangled. If we put $k=1$ and in the absence of the Stark shift corresponding to the negativity in the two atom TCM obtained by Ref.[13]. From Fig. 5b, i.e. $R=1\left(\beta_{1}=\beta_{2}\right)$, we find that the Stark shift leads to an increase in the value of entanglement at $(\tau=n \pi)$ and when $\tau=4 \pi$ we get the maximum entanglement. In Fig. 5c, d, we show the cases in which the two levels have unequal Stark shifts ( $R<1$, in Fig. 5 c $R=0.6$ while in Fig. $5 \mathrm{~d} R=0.3$ ). We see that the Stark shift leads to a decrease in the values of the maximum entanglement and the evolution period of the atom entropy decreases with the parameter $R$. Aloso, if we take the two atoms in ground state $|-,-\rangle$ we get the same results for the two atoms in exited state. But if the two atoms are initially prepared in $|+,-\rangle$ see Fig. 6 the maximum value of entanglement is decreased and the negativity evolves with a period $\pi / 2$. The entanglement returns to increase in the case of $\beta_{1}=\beta_{2}$ and for $R=0.6, R=0.3$ (see Fig. 6c,d) the situation is changed because we can observe that the stark shift leads to a decrease in the amplitudes of the maximum value of the negativity and finally the state returns to entangled state with periodicity is lost. 

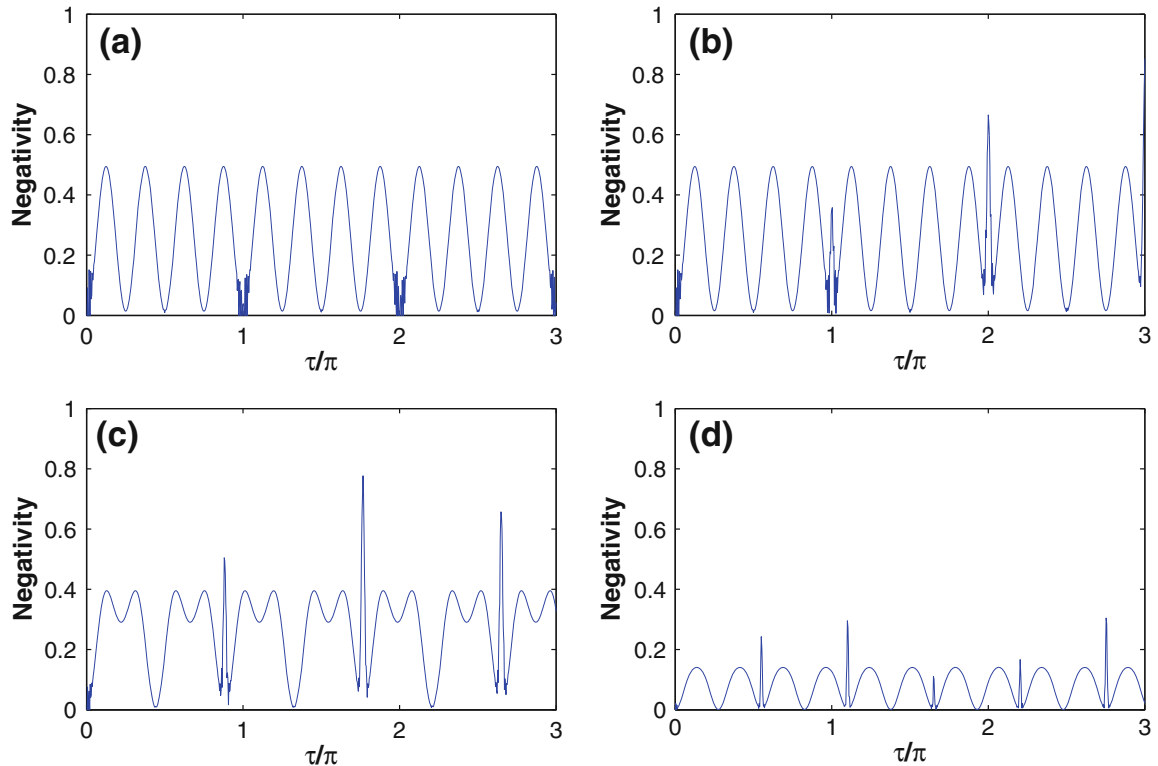

Fig. 5 The time evolution of the negativity for the two atoms are prepared initially in the exited state and the field in a coherent state for two-photon $(k=2)$ with initial mean photon number $\bar{n}=100$ for various values of the Stark shift parameter $R$ : a neglect of the Stark shift $\mathbf{b} R=1\left(\beta_{1}=\beta_{2}\right) \mathbf{c} R=0.6 \mathbf{d} R=0.3$
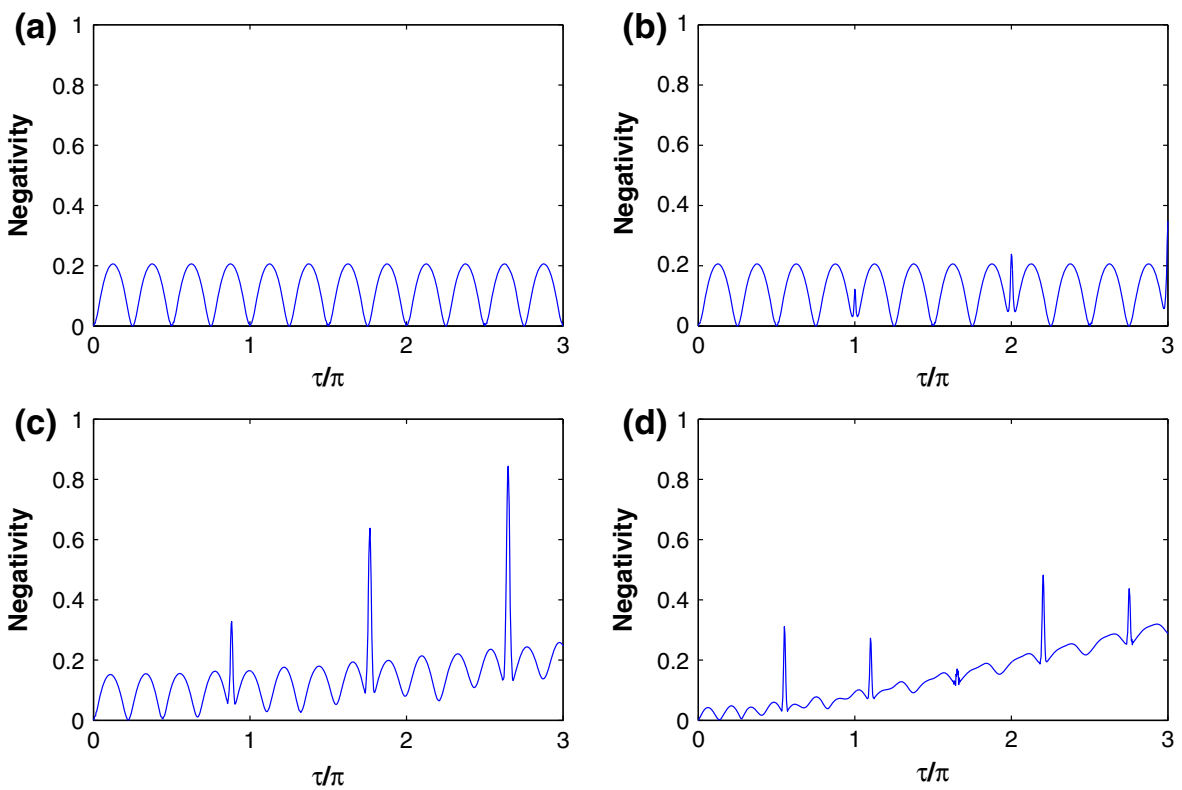

Fig. 6 The time evolution of the negativity for the two atoms are prepared initially in $|+,-\rangle$ state and the field in a coherent state for two-photon $(k=2)$ with initial mean photon number $\bar{n}=100$ for various values of the Stark shift parameter $R$ : a neglect of the Stark shift $\mathbf{b} R=1\left(\beta_{1}=\beta_{2}\right) \mathbf{c} R=0.6 \mathbf{d} R=0.3$ 


\section{Conclusions}

In this paper, we have presented an analytic solution for the system of two 2-level atoms interacting with a multi-photon single-mode field, taking into account the Stark shifts. The entanglement is found to appear in the model for some initial atomic states and coherent field input with different values of the parameter of stark shift. We have investigated the influence of the Stark shift on the entanglement by using various measures such as the von Neumann entropy, tangles and negativity. It is found that, for small values of the parameter of Stark shift $(R<1)$, the Stark shift leads to a decrease of the values of the maximum atomic entropy and the evolution period of the atomic entropy. Also it has been shown that the tangle $\tau_{F\left(A_{1} A_{2}\right)}$ as a measure of entanglement has typical information on the purity as the entropy. We have shown that for the tangle $\tau_{A_{1}\left(A_{2} F\right)}$ a large change occurs in the behavior where we note an increase in the fluctuations number and consequently more periods at different intervals. The contribution to the purity loss of the global system is from the interaction between atom and field. The tangle is used to measure the coherence loss of the atomic and field states. Furthermore we discussed the negativity as a measure of entanglement degree and we found that for decreasing the value of the parameter of the Stark shift the value of entanglement is decreased for $R<1$, while for $R=1$ we get the maximum entanglement.

Open Access This article is distributed under the terms of the Creative Commons Attribution Noncommercial License which permits any noncommercial use, distribution, and reproduction in any medium, provided the original author(s) and source are credited.

\section{References}

1. Plenio, M.B., Vedral, V.: Entanglement measures and purificationprocedures. Phys. Rev. A 57, 1619 (1998)

2. Phoenix, S.J.D., Kinght, P.L.: Periodicity, phase, and entropy in models of two-photon resonance. Ann. Phys. 186, 381 (1988)

3. Cirac, J.I., Zoller, P.: Quantum computations with cold trapped ions. Phys. Rev. Lett. 74, 4091 (1995)

4. Bennett, C.H., Brassard, G., Crepeau, C., Jozsa, R., Peres, A., Wootters, W.K.: Teleporting an unknown quantum state via dual classical and Einstein-Podolsky-Rosen channels. Phys. Rev. Lett. 70, 1895 (1993)

5. Braunstein, S.L., Kimble, H.J.: Dense coding for continuous variables. Phys. Rev. A 61, 042302 (2000)

6. Bennett, C.H., Divincenzo, D.P.: Quantum information and computation. Nature 404, 247 (2000)

7. DeVoe, R.G., Brewer, R.G.: Observation of Superradiant and Subradiant Spontaneous Emission of Two Trapped Ions. Phys. Rev. Lett. 76, 2049 (1996)

8. Turchette, Q.A., Wood, C.S., King, B.E., Myatt, C.J., Leibfried, D., Itano, W.M., Monroe, C., Wineland, D.J.: Deterministic entanglement of two trapped ions. Phys. Rev. Lett. 81, 3631 (1998)

9. Hagley, E., Maitre, X., Nogues, G., Wunderlich, C., Brune, M., Raimond, J.M., Haroche, S.: Generation of Einstein-Podolsky-Rosen Pairs of Atoms. Phys. Rev. Lett. 79, 1 (1997)

10. Tavis, M., Cummings, F.W.: Exact Solution for an N-MoleculeRadiation-Field Hamiltonian. Phys. Rev. 170, 379 (1968)

11. Nielsen, M.A., Chuang, I.L.: Quantum computation and quantum information. Cambridge University Press, Cambridge (2000)

12. Alber, G., Beth, T., Horodecki, M., Horodecki, P., Horodecki, R., Rötteler, M., Weinfurter, H., Werner, R., Zeilinger, A.: An Introduction to Basic Theoretical Concepts and Experiments. Quantum Information. Springer, Berlin (2001) 
13. Tessier, T.E., Deutsch, I.H., Delgado, A.: Entanglement sharing in the two-atom Tavis-Cummings model. Phys. Rev. A 68, 062316 (2003)

14. Liu, T.-K., Wang, J.-S., Feng, J., Zhan, M.-S.: Entropy evolution properties in a system of two entangled atoms interacting with light field. Chin. Phys. Soc. 14, 536 (2005)

15. Jin, L.-J., Fang, M.F.: Entanglement in a system of two two-level atoms interacting with a single-mode field. Chin. Phys. Soc. 15, 2012 (2006)

16. Ma, J.-M., Jiao, Z.-Y., Li, N.: Entropy and entanglement of a single-mode vacuum field interacting with a $\Xi$ type three-level atom with detuning. Int. J. Theor. Phys. 47, 350 (2008)

17. Jiao, Z.-Y., Ma, J.-M., Li, N., Xia, F.: Entropy of the binomial field interacting with the two entangled atoms. Int. J. Theor. Phys. 47, 2841 (2008)

18. Wootters, W.K.: Entanglement of formation of an arbitrary state of two qubits. Phys. Rev. Lett. 80, 2245 (1998)

19. Hill, S., Wootters, W.K.: Entanglement of a pair of quantum bits. Phys. Rev. Lett. 78, 5022 (1997)

20. Peres, A.: Separability criterion for density matrices. Phys. Rev. Lett. 77, 1413 (1996)

21. Horodecki, P.: Separability criterion and inseparable mixed states with positive partial transposition. Phys. Lett. A 232, 333 (1997)

22. Angelo, R.M., Furuya, K., Nemes, M.C., Pellegrino, G.Q.: Recoherence in the entanglement dynamics and classical orbits in the N-atom Jaynes-Cummings model. Phys. Rev. A 64, 043801 (2001)

23. Yu, T. , Eberly, J.H.: Quantum open system theory: bipartite aspects. Phys. Rev. Lett. 97, 140403 (2006)

24. Yu, T., Eberly, J.H.: Evolution from entanglement to decoherence of bipartite mixed "X" states. Quantum Inf. Comput. 7, 459 (2007)

25. Yu, T., Eberly, J.H.: Sudden death of entanglement: classical noise effects. Opt. Commun. 264, 393 (2006)

26. Jex, I., Matsuka, K.M.: Phase of the field in the interaction with two two-level atoms. Quantum Opt. 5, 275 (1993)

27. Knöll, L., Orowski, A.: Distance between density operators: applications to the Jaynes-Cummings model. Phys. Rev. A 51, 1622 (1995)

28. Yoo, H.I., Eberly, J.H.: Dynamical theory of an atom with two or three levels interacting with quantized cavity fields. Phys. Rep. 118, 239 (1985)

29. Buzek, V., Moya-Cessa, H., Knight, P.L., Phoenix, S.J.: Schrödinger-cat states in the resonant JaynesCummings model: Collapse and revival of oscillations of the photon-number distribution. Phys. Rev. A 45, 8190 (1992)

30. Rungta, P., Buzek, V., Caves, C.M., Hillery, H., Milburn, G.J.: Universal state inversion and concurrence in arbitrary dimensions. Phys. Rev. A 64, 042315 (2001)

31. Coffman, V., Kundu, J., Wootters, W.K.: Distributed entanglement. Phys. Rev. A 61, 052306/1 (2000)

32. Scheel, S., Eisert, J., Knight, P.L., Plenio, M.B.: Hot entanglement in a simple dynamical model. J. Mod. Optics 50, 881 (2003)

33. Tang-Kun, L., Ji-Suo, L., Jain, F., Ming-Sheng, Z.: Entropy evolution properties in a system of two entangled atoms interacting with light field. Chin. Phys. 14, 536 (2005) 